\title{
INDEX TO VOLUME XVIII.
}

Archibald, R. C. See Reviews, under Sommerville.

Bateman, H. On a Set of Kernels Whose Determinants Form a Sturmian Sequence, 179.

Bader, G. N. See Reviews, under Fouët.

Bernstein, B. A. See Leuschner, A. O.

Burss, G. A. A New Proof of the Existence Theorem for Implicit Functions, 175 .

- See Reviews, under Eisenhart.

Bôcher, M. The Published and Unpublished Work of Charles Sturm on Algebraic and Differential Equations, 1.

BolzA, O. A Generalization of Lindelöf's Theorems on the Catenary, 107.

Brown, E. W. See Reviews, under Andoyer, Annuaire, Darwin.

Camp, B. H. Series of Laplace's Functions, 236.

Chapman, S. A Note on the Theory of Summable Integrals, 111.

Clements, G. R. Implicit Functions Defined by Equations with Vanishing Jacobian, 451.

CoLe, F. N. Reports of Meetings of the American Mathematical Society: Eighteenth Summer Meeting, 51; October Meeting, 159; Eighteenth Annual Meeting, 213; February Meeting, 327; April Meeting, 485.

Coolidge, J. L. See Reviews, under Veblen.

Dickson, L. E. See Reviews, under Heath.

Dumont, E. See Reviews, under Dumont.

Eisenhart, I. P. See Reviews, under Bianchi.

Emcr, A. See Reviews, under Crelier.

Fite, W. B. Irreducible Homogeneous Linear Groups of Order $p^{m}$ and Degree $p$ or $p^{2}, 117$.

Ginlespie, D. C. Definite Integrals Containing a Parameter, 379.

Haskins, C. N. See Reviews, under Holton.

Hayashi, T. An Expression for the General Term of a Recurring Series, 191.

Hurwitz, W. A. Note on Mixed Linear Integral Equations, 291.

International Commission on the Teaching of Mathematics. Committee Report: Graduate Work in Mathematics in Universities and in Other Institutions of Like Grade in the United States, 122.

KellogG, O. D. The Fifth Regular Meeting of the Southwestern Section, 232.

- See Reviews, under Weber.

Keyser, C. J. A Sensuous Representation of Paths that Lead from the Inside to the Outside of an Ordinary Sphere in Point Space of Four Dimensions without Penetrating the Surface of the Sphere, 18.

Laves, K. See Reviews, under Andoyer.

Lefschetz, S. On the $V_{3}{ }^{3}$ with Five Nodes of the Second Kind in $S_{4}, 384$.

Lennes, N. J. A Direct Proof of the Theorem that the Number of Terms in the Expansion of an Infinite Determinant is of the Same Potency as the Continuum, 22.

- A Necessary and Sufficient Condition for the Uniform Convergence of a Certain Class of Infinite Series, 66, 200. 
See Reviews, under Dumont, Young.

Leuschner, A. O., and Bernstein, B. A. Note on the Graphical Solutions of the Fundamental Equations in the Short Methods of Determining Orbits, 294.

Lipke, J. See Reviews, under Fleck, Lind.

Longley, W. R. Proof of a Theorem due to Picard, 493.

Lythe, See Reviews, under Young.

McKelvey, J. V. See Reviews, under Hawkes.

MacMillan, W. D. See Reviews, under Smith.

Miller, G. A. Note on the Maximal Cyclic Subgroups of a Group of Order $p^{m}, 189$.

See Reviews, under Muir.

Mitcheld, H. H. Note on Collineation Groups, 146.

Moore, C. L. E. Surfaces in Hyperspace Which Have a Tangent Line with Three-Point Contact Passing through Each Point, 284.

Moore, E. H. On the Foundations of the Theory of Linear Integral Equations, 334.

Moritz, R. E. On the Cubes of Determinants of the Second, Third, and Higher Orders, 182.

NeIkIRK, L. I. See Reviews, under Müller.

Ponzer, E. W. See Reviews, under Siddons, Timerding.

Owens, F. W. See Reviews, under Schur, Slaught.

Putnam, T. M. Reports of Meetings of the San Francisco Section: October Meeting, 166; April Meeting, 448.

SAFFord, F. H. An Identical Transformation of the Elliptic Element in the Weierstrasss Form, 279.

Schweitzer, A. R. On a Functional Equation, 299.

Shaw, J. B. See Reviews, under Appell, Friedel, Whitehead.

Sladght, H. E. Reports of Meetings of the Chicago Section: Winter Meeting, 269; April Meeting, 431.

SNYDER, V. The Carlsruhe Meeting of the German Mathematical Society, 169.

- See Reviews, under Séférian.

WARner, C. F., See Reviews, under Holton.

Webster, A. G. On a New Mixed Problem of the Partial Differential Equation of Telegraphy, 244.

Wilczynski, E. J. See Reviews, under Cohen.

Wilson, E. B. See Reviews, under Chwolson, Hosmer, Jouguet, Pareto.

Young, J. W. See Reviews, under Lebon.

\section{Reviews.}

Andoyer, H. Nouvelles Tables trigonométriques fondamentales, E. W. Brown, 365.

Cours d'Astronomie (deuxième édition), K. Laves, 517.

Annuaire du Bureau des Longitudes pour l'An 1912, E. W. Brown, 368. Appell, P., et Dautheville, S. Précis de Mécanique rationelle, J. B. SHAw, 311.

Bianchi, L. Vorlesungen über Differentialgeometrie (Deutsche Uebersetzung von M. Lukat, zweite Auflage), L. P. Eisenrart, 411. 
Chwolson, O. D. Traité de Physique (traduit par E. Davaux), Vols. I-II, E. B. WILSON, 497.

Cohen, A. Introduction to the Lie Theory of One-Parameter Groups with Applications to the Solution of Differential Equations, E. J. WILCZYNSKI, 514.

Crelier, L. Systèmes cinématiques, A. Емсн, 316.

Darwin, G. H. Scientific Papers, Vols. III-IV, E. W. Brown, 456.

Dautheville, S. See Appell, P.

Davaux, E. See Chwolson, O. D.

Dumont, E. Arithmétique générale, N. J. Lennes, 307,511. E. Dumont, 508.

Eisenhart, L. P. Differential Geometry of Curves and Surfaces, G. A. BLISS, 145.

Fleck, A., Maennschen, P., Perron, O. Vermeintliche Beweise des Fermatschen Satzes, J. LIPKE, 194.

Fouët, E. A. Leçons élémentaires sur la Théorie des Fonctions analytiques (deuxième édition), G. N. BAUER, 30.

Friedel, G. Leçons de Cristallographie, J. B. Shaw, 364.

Hawkes, H. E., Luby, W. A., and Touton, F. C. First Course in Algebra, J. V. MCKelveY, 199.

Heath, T. L. Diophantus of Alexandria (second edition), L. E. Dickson, 82.

Holton, E. E. Shop Mathematics, C. N. Haskins, 137, 201, 306; C. F. WARNER, 303.

Hosmer, G. L. Azimuth, E. B. WiLson, 144.

Jouguet, E. Lectures de Mécanique, deuxième partie, E. B. Wilson, 32.

Lebon, E. Emile Picard, Paul Appell; Biographie, Bibliographie analytique des Ecrits, J. W. YounG, 193.

Lennes, N. J. See Slaught, H. E.

Lind, B. Ueber das letzte Fermatsche Theorem, J. LiPkE, 194.

Longley, W. R. See Smith, P. F.

Luby, W. A. See Hawkes, H. E.

Lukat, M. See Bianchi, L.

Maennschen, P. See Fleck, A.

Muir, T. Theory of Determinants in the Historical Order of Development, Vol. II, G. A. Miller, 512.

Müller, E. Abriss der Algebra der Logik, Teile I-II, L. I. NeIKIRK, 515.

Pareto, V. Manuel d' Economie Politique, E. B. Wilson, 462.

Perron, O. See Fleck, A.

Russell, B. See Whitehead, A. N.

Schur, F. Grundlagen der Geometrie, F. W. Owens, 309.

Séférian, A. Notice sur le Système des six Coordonnées homogènes d'une Droite et sur les Eléments de la Théorie des Complexes linéaires, V. SNYDER, 84.

Siddons, A. W., and Vassall, A. Practical Measurements, E. W. Ponzer, 144.

Slaught, H. E., and Lennes, N. J. Solid Geometry, F. W. Owens, 198.

Smith, P. F., and Longley, W. R. Theoretical Mechanics, W. D. MACMillan, 84.

Sommerville, D. M. Y. Bibliography of Non-Euclidean Geometry, R. C. ARchi batd, 254. 
Timerding, H. E. Die Mathematik in den physikalischen Lehrbüchern, Band III, Heft 2, E. W. Ponzer, 142.

Touton, F. C. See Hawkes, H. E.

Vassall, A. See Siddons, A. W.

Veblen, O., and Young, J. W. Projective Geometry, Vol. I, J. L. CooLIDGE, 70.

Weber, H. Die partiellen Differentialgleichungen der mathematischen Physik, Erster Band (fünfte Auflage), O. D. Kelloga, 87.

Whitehead, A. N., and Russell, B. Principia Mathematica, Vol. I, J. B. Shaw, 386.

Young, J. W. Lectures on Fundamental Concepts of Algebra and Geometry, E. B. LYTLE, 362. See Veblen, $O$.

Young, W. H., and G. C. The Theory of Sets of Points, N. J. Lennes, 24.

Corrections, 33, 200.

Index of Volume, 535 .

New Publications, 43, 97, 154, 204, 263, 320, 373, 425, 480, 521.

Notes, 34, 89, 147, 202, 258, 317, 369, 419, 474, 519.

Papers read before the Society and Pubsequently Published, TwentyFirst Annual List of, 528.

\section{Notes AND OTher Items.}

Academies, Associations, Congresses, and Societies:

American Mathematical Society: Administration, 160; Annual Meeting, 147, 432, 485; Annual Register, 147, 160; Chicago Section, 147, 258; Election of Officers, 214; Library, 214, 327; New Members Admitted, 51, 159, 213, 327, 485; Statistics, 214; Summer Meeting, $52,159,369$; Transactions, $147,258,419$.

Associations for the Advancement of Science: British, 34, 370; French, 34, 370; Italian, 34, 148.

Associations of Teachers of Mathematics: International Commission on the Teaching of Mathematics, 36, 149, 260, 318, 370, 370; Middle States and Maryland, 371; National Committee of Fifteen on Geometry Syllabus, 35, 318.

Belgian Academy, 260, 420; Deutsche Mathematiker-Vereinigung, 519; Edinburgh Mathematical Society, 202, 259, 317, 370, 519; Fifth International Congress of Mathematicians, 34, 36, 318, 420, 474; Göttingen Academy, 520; Jablonowski Society, 89; London Mathematical Society, 202, 259, 261, 317, 369, 419, 475; Mathematical Association, 259; Naples Royal Society, 35; Paris Academy, 35, 203, 318, 420; Royal Society, 203; Russian Congress of Mathematicians, 202, 420; Scandinavian Congress of Mathematicians, 34 .

Books, Announcements of New, 148, 148, 149, 202, 203, 260, 318, 370, $519,519,520$.

Catalogues of Books, Models, etc., 153, 203, 319, 319, 371, 372, 419, 521.

Doctorates in Mathematics, American, 36; French, 423.

Journals:

American Journal of Mathematics, 89, 259, 419; Annals of Mathematics, 34, 148, 259, 369; Tôhoku Mathematical Journal, 148; Transactions of the American Mathematical Society, 147, 258, 419; Vector, 202. 
Papers and Communications Presented to the Society, Authors:

Baker, R. P., 433.

Beal, F. W., 52.

Bennett, E. R., 52.

Bernstein, B. A., 166, 448, 448.

Bill, E. G., 270, 432.

Birkhoff, G. D., 53, 53, 486, 486.

Blichfeldt, H. F., 166, 448.

Bliss, G. A., 53, 160.

Bowden, J., 52, 53 ,

Brown, E. W., 160.

Buck, T., 448.

Burgess, H. T., 53.

Cajori, F., 52, 433.

Camp, B. H., 160, 486.

Carmichael, 160, 160, 160, 232, 270, 270, 433, 433 .

Castle, E. W., 52.

Chittenden, E. W., 433.

Coble, A. B., 328.

Curtiss, D. R., 432.

Denton, W. W., 433.

Dickson, L. E., 270, 270, 270.

Dines, L. L., 160.

Dodd, E. L., 433.

Dresden, A., 270, 270.

Dunkel, O., 232.

Eiesland, J., 215.

Eisenhart, L. P., 52, 216, 216.

Emch, A., 52, 270, 432.

Ernsberger, I., 232.

Field, P., 270.

Fite, W. B., 216.

Frizell, A. B., 233.

Glenn, O. E., 215.

Glover, J. W., 433.

Gronwall, T. H., 433, 433.

Haskins, C. N., 216.

Hedrick, E. R., 232, 233.

Hildebrandt, T. H., 433.

Hodgson, J. E., 328.

Howland, L. A., 215, 486.

Huntington, E. V., 160.

Hurwitz, W. A., 52, 52.

Ingold, L., 232, 270.

Jackson, D., 328, 328, 486.

James, G. O., 233, 432.

Joffe, S. A., 328.

Karpinski, L. C., 52, 433.

Kasner, E., 160, 215.

Kellogg, O. D., 232.

Lamond, J. K., 486.

Landau, E., 52.
Laves, K., 432.

Lefschetz, S., 52, 53, 232, 328, $432,448,486$.

Lennes, N. J., 160, 486, 486.

Leuschner, A. O., 166.

Longley, W. R., 486.

Manning, W. A., 166.

Marshall, E. R., 486.

Miles, E. J., 328.

Miller, G. A., 52, 52, 166, 215, 270, 328, 433.

Mitchell, H. H., 160, 328.

Moore, C. L. E., 215, 215, 486, 486.

Moore, C. N., 215.

Moore, E. H., 433.

Moore, R. L., 215, 486.

Moulton, F. R., 270, 433.

Osgood, W. F., 328.

Phillips, H. B., 215, 486, 486.

Pitcher, A. D., 486.

Powers, R. E., 160.

Ranum, A., 52, 215.

Richardson, R. G. D., 215.

Roever, W. H., 232, 432, 432, 432.

Root, R. E., 270.

Rowe, J. E., 52, 215, 215, 328.

Schweitzer, A. R., 160, 486, 486.

Sharpe, F. R., 215.

Shaw, J. B., 270.

Siceloff, L. P., 160, 486.

Silverman, L. L., 216, 216.

Slocum, S. E., 215.

Smith, W. M., 215.

Snyder, V., 53.

Spunar, V. M., 433.

Stager, H. W., 166.

Stouffer, E. B., 433.

Urner, S. E., 328.

Van Benschoten, A. L., 215.

Vandiver, H. F., 270.

Veblen, O., 53, 486.

Wahlin, G. E., 432.

Webster, A. G., 53, 53, 53.

White, M. B., 232.

Whitney, A. W., 166.

Whittemore, J. K., 432.

Wilczynski, E. J., 270, 432.

Williams, K. P., 328.

Wilson, E. B., 215.

Young, J. W., 216, 216.

\section{Personal Notes:}

Agard, H. L., 36; Amsler-Laffon, J.,.424; Andoyer, H., 261; Appell,

P., 479; d'Arcais, F., 41; Arzelá, M. C., 480; Ashcraft, T. B., 36.

Bacon, C. I., 36; Baker, R. P., 36; Baldus, R., 260, 261; Barney, 
I., 36; Barnhart, C. A., 479; Bateman, H., 480; Bates, W. H., 36; Beal, F. W., 36; Belot, E., 34, 370; Bernstein, S., 260; Biddle, A. D., 36, 204; Bill, E. G., 96, 372, 424; Birkhoff, G. D., 148, 424; Bliss, G. A., 37; Bôcher, M., 148, 475; Boehm, F., 153; Borel, E., 34, 420, 475; Boss, L., 35; Boyd, P. P., 36, 479; Brenke, W. C., 519; Brooke, W. E., 521 ; Brown, E. W., 34, 152, 420, 475; Buchanan, D., 36, 42; Buchanan, H. E., 42, 96; Burgatti, P., 262; Burgess, A. G., 202; Burgess, R. W., 424.

Cajori, F., 35, 520; Callendar, H. L., 370; Camp, B. H., 36; Cantor, G., 41; Carmichael, R. D., 36; Castelnuovo, G., 34; Chatenet, L., 260; Chazy, E., 260; Chittenden, M. W., 479; Chrystal, G., 203, 262, 372; Coates, W. M., 319; Cohen, A., 203; Colpitts, E. C., 262; Conner, J. R., 480; Courant, R., 478; Cowrie, P., 202; Cox, L. C., 97, 480; Cresse, G. H., 42; Czuber, E., 479.

Darboux, G., 261, 423; Darwin, G. H., 203; Davis, E. W., 519; Dedekind, R., 41; Demoulin, A., 479; Denton, W. W., 479; Deruyts, J., 203; Dines, C. R., 42; Dini, U., 41 ; Drach, J., 318; Dunning, A. B., 96; Duval, E. P. R., 42; v. Dyck, W., 519.

Eddy, H. T., 520; Eisenhart, L. P., 148; Engel, F., 153; Enriques, F., 261,475 .

Faber, G., 261; Fairon, E., 203, 260; Fejér, L., 40; Filon, L. N. G., 424; Fine, H. B., 479; Fischer, C. A., 424; Fischer, E., 95; Francis, W. A., 204; Frizell, A. B., 262; Fuchs, R., 96; Furtwängler, P., 153.

Gaba, M., 41; Gable, G. D., 42; Galajikian, A. S., 479; Galitzin, B., 475; Gans, R., 95; Gauss, C. F., 95; Gavett, G. I., 204; Geckeler, O. T., 96; Giambelli, Z., 262; Grünwald, J., 97; Guccia, G. B., 41, 423. Hadamard, J., 260, 520; Hancock, E. L., 97; Hassler, J. O., 42; Hatzidakis, N., 153; Hawkes, H. E., 204; Hawkesworth, A. S., 42; Hayashi, T., 148; Haynes, A. E., 480; H'Doubler, F. T., 96; Hennequin, M., 40; Henriques, H., 262; Hilbert, D., 153; Hobson, E. W., 203; Holman, W. F., 521; Hostinsky, B., 479.

Ingold, L., 424 ; Isely, L., 41.

Jackson, D., 95; Janet, M. P., 260; Jones, G. W., 153; Jones, J. L., 37, 153; Jordan, C., 520; Jordan, H. E., 42; Jouguet, E., 35.

Klein, F., 95, 318; Kneser, A., 34, 420; Knopp, K., 261; v. Koch, H., 96; Kommerell, K., 261; König, R., 95; Kowalewski, G., 478; Krathwohl, W. C., 42; Kruppa, E., 262; Kutta, W., 424.

Lamb, H., 261; Landau, E. G. H., 34, 420, 475; Larmor, J., 34, 41, 420, 475; Láska, W., 41; Lauricella, G., 261; Lefschetz, S., 37, 96; Lemoine, E., 35, 424; Levi-Civita, T., 41; Lindsay, L., 37; Lockner, J. S., 204; London, F., 152; Lovitt, W. V., 480.

McEwen, G. F., 479; MacGregor, H.'H., 42; MacInnes, C. R., 204; Majcen, G., 96; Maillet, E., 204; Marriott, W. R., 37; Mason, M., 37; Mendenhall, W. O., 37; Mertens, F., 97; Miller, A. L., 480; Miller, G. A., 479; Mittag-Leffler, G., 96; Montesano, D., 41; Montgomery, W. J., 37; Moore, R. L., 42; Morgan, F. M., 424; Morley, R. K., 479;

Mott, H. W., 42.

Neumann, E., 424; Noether, F., 262; Nusl, F., 261.

d'Ocagne, M., 260; O'Shaughnessy, L., 37.

Pfeiffer, F., 479; Picard, E., 203; Pierpont, J., 519; Pitcher, A. D., 37, 41; Plancherel, M., 40; Pollack, V., 372.

Rateau, A., 35; Reddick, H. W., 37; Reid, L. W., 42; Reynolds, O., 424; Riesz, M.; 372; Rietz, H. L., 96; Rorer, J. T., 204; Rowe, J. E., 42,424 .

Salkowsky, E., 96 ; v. Sanden, H., 95; Schimmack, R., 95; Schirmer, G., 260; Schleiermacher, L., 95; Schmidt, E., 41; Schoenflies, A., 96; Schrutka, L., 520; Schubert, H., 97; Schulof, L., 35; Shattuck, S. W., 480; Siceloff, I. P., 424; Sisam, C. H., 520; Slaught, H. E., 41; Sles- 
zynski, J., 372; Smith, A. G., 41; Smith, P. F., 204; Smith, W. E., 42; Snyder, V., 204, 520, 520; Sommerville, D. M. Y., 202; Stouffer, E. B., 37, 96; Ströle, H., 262; Study, E., 153.

Tannery, J., 35; Tedone, O., 41; Thomson, J. J., 261; Timpe, A., 262; Tracey, J. I., 480; Traynard, E., 203; Turner, H. H., 34.

Urner, S. E., 37.

Van der Vries, J. N., 42; Vassilief, A., 420; Veblen, O., 148; Vessiot, E., 260; Voigt, W., 95; Volterra, V., 261; Voss, A., 261.

Webber, W. P., 37; Weld, L. G., 41, 153; Wheeler, J. J., 42; White, M. B., 37; White, W. H., 34, 420, 175; Whitehead, A. N., 96; Whitmore, J., 204; Whittaker, E. T., 372; Wieghardt, K., 262; Wilson, E. B., 148; Wilson, W. A., 37; Winger, R. M., 480.

Yanney, B. F., 96; Yeaton, C. H., 97.

Prizes:

Zermelo, E., 520; Zoretti, E., 41 .

Belgian Academy, 260, 420; De Morgan, 261; Elsa Neumann, 35; Göttingen University, 95; Göttingen Academy, 520; Jablonowski Society, 89, 424; London Mathematical Society, 261; Naples Academy, 35; Paris Academy, 35, 203, 318, 420; Royal Society, 203; Schirmer, 260.

Universities and Technical Schools:

Aachen, 150.

Berlin, 90, 150, 420.

Bologna, 38.

Bonn, $91,421$.

Breslau, 91, 150 .

Brunswick, 150.

Catania, 38.

Chicago, 475, 478.

Clark, 476.

Columbia, 422, 478.

Cornell, 422, 478.

Danzig, 151.

Darmstadt, 151.

Delft, 151.

Dresden, 151 .

Erlangen, 91.

Freiburg, 91.

Genoa, 38.

Giessen, 91.

Göttingen, 92, 421.

Greifswald, 92.

Halle, 92.

Hanover, 152.

Harvard, 37, 476.

Illinois, 476 .

Indiana, 477, 478.

Jena, 92.

Johns Hopkins, 477.

Karlsruhe, 152.

Kiel, 93.

Königsberg, 93.

Leipzig, 93.

Marburg, 93.

Massachusetts Institute of Technology, 90, 477.

Michigan, 520.

Munich, 94, 371.

Naples, 39.

Padua, 39.

Palermo, 39.

Paris, 149.

Pavia, 39.

Pisa, 39.

Princeton, 477.

Rome, 40.

Rostock, 94.

Strassburg, 94, 372.

Stuttgart, 152.

Tübingen, 94.

Turin, 40.

Würzburg, 95. 
